\title{
THE STRONG LAW OF LARGE NUMBERS FOR EXTENDED NEGATIVELY DEPENDENT RANDOM VARIABLES
}

\author{
YIQING CHEN,*** University of Liverpool \\ ANYUE CHEN,${ }^{*}$ Xi'an Jiaotong-Liverpool University \\ KAI W. NG, ${ }^{* * *}$ The University of Hong Kong
}

\begin{abstract}
A sequence of random variables is said to be extended negatively dependent (END) if the tails of its finite-dimensional distributions in the lower-left and upper-right corners are dominated by a multiple of the tails of the corresponding finite-dimensional distributions of a sequence of independent random variables with the same marginal distributions. The goal of this paper is to establish the strong law of large numbers for a sequence of END and identically distributed random variables. In doing so we derive some new inequalities of large deviation type for the sums of END and identically distributed random variables being suitably truncated. We also show applications of our main result to risk theory and renewal theory.
\end{abstract}

Keywords: Asymptotics; Borel-Cantelli lemma; lower/upper extended negative dependence; renewal counting process; strong law of large numbers; truncation

2010 Mathematics Subject Classification: Primary 60F15

Secondary 60K05

\section{Introduction}

Random variables $X_{k}, k=1, \ldots, n$, are said to be lower extended negatively dependent (LEND) if there is some $M>0$ such that, for all $x_{k}, k=1, \ldots, n$,

$$
\operatorname{Pr}\left(\bigcap_{k=1}^{n}\left(X_{k} \leq x_{k}\right)\right) \leq M \prod_{k=1}^{n} \operatorname{Pr}\left(X_{k} \leq x_{k}\right) ;
$$

they are said to be upper extended negatively dependent (UEND) if there is some $M>0$ such that, for all $x_{k}, k=1, \ldots, n$,

$$
\operatorname{Pr}\left(\bigcap_{k=1}^{n}\left(X_{k}>x_{k}\right)\right) \leq M \prod_{k=1}^{n} \operatorname{Pr}\left(X_{k}>x_{k}\right) ;
$$

and they are said to be extended negatively dependent (END) if they are both LEND and UEND. A sequence of infinitely many random variables $\left\{X_{k}, k=1,2, \ldots\right\}$ is said to be LEND, UEND, or END if, for each positive integer $n$, the random variables $X_{k}, k=1, \ldots, n$,

Received 10 February 2010; revision received 23 June 2010.

* Postal address: Department of Mathematical Sciences, University of Liverpool, Liverpool, L69 7ZL, UK.

** Email address: yiqing.chen@liv.ac.uk

*** Postal address: Department of Statistics and Actuarial Science, The University of Hong Kong, Pokfulam Road, Hong Kong. 
are LEND, UEND, or END, respectively. Sometimes we need to specifically mention the dominating constant $M$ associated with the LEND, UEND, or END structures.

When $M=1$, inequalities (1.1) and (1.2) describe lower and upper negative dependencies, respectively. The concept of negative dependence has been extensively investigated since it was proposed in Ebrahimi and Ghosh (1981) and Block et al. (1982). In particular, Matuła (1992) established the strong law of large numbers for pairwise negatively dependent random variables. A key step of Matuła's (1992) derivation is that, by Hoeffding's identity, the covariance of two negatively dependent random variables, being suitably truncated, is nonpositive. Hence, the pairwise negative dependence greatly prevents the partial sums from diverging to infinity. In general, this implication is not true for END random variables. Therefore, the approach we shall employ in this paper is essentially different from Matuła's (1992). Recent developments of the strong law of large numbers for negatively dependent random variables can be found in Bingham and Nili Sani (2004), Gerasimov (2009), and Baek et al. (2009), among others.

As a natural generalization of negative dependence, the concept of END was proposed in Liu (2009) and further promoted in Chen et al. (2010) in the study of precise large deviations. The END structure covers all negative dependence structures and, more interestingly, it covers certain positive dependence structures. A sufficient condition for LEND or UEND is given in Lemma 2.1 below. In particular, by this lemma, every $n$-dimensional FarlieGumbel-Morgenstern (FGM) distribution describes a specific END structure. Recall that an $n$-dimensional FGM distribution has the form

$$
F_{1, \ldots, n}\left(x_{1}, \ldots, x_{n}\right)=\left(\prod_{k=1}^{n} F_{k}\left(x_{k}\right)\right)\left(1+\sum_{1 \leq i<j \leq n} a_{i j} \bar{F}_{i}\left(x_{i}\right) \bar{F}_{j}\left(x_{j}\right)\right),
$$

where $F_{k}=1-\bar{F}_{k}, k=1, \ldots, n$, are corresponding marginal distributions and $a_{i j}$ are real numbers chosen such that $F_{1, \ldots, n}$ is a proper $n$-dimensional distribution. We refer the reader to Kotz et al. (2000, Chapter 44.13) for a general account on multivariate FGM distributions. Owing to its transparent structure and great flexibility of adjusting dependence strength, this family of multivariate distributions is often used in modelling dependent investment returns in finance or dependent claim sizes in insurance; see, for example, Tang and Vernic (2007) and Cossette et al. (2008), among many others. Hashorva (2001) established limit theorems for a sequence of random variables with FGM finite-dimensional distributions.

Our main result is the following.

Theorem 1.1. Let $\left\{X_{k}, k=1,2, \ldots\right\}$ be a sequence of END random variables with common distribution $F$. Denote by $S_{n}$ its $n$th partial sum, $n=1,2, \ldots$. Then $S_{n} / n \stackrel{\text { a.s. }}{\rightarrow} \mu$ as $n \rightarrow \infty$ for some real number $\mu$ if and only if $\mathrm{E}\left|X_{1}\right|<\infty$, and for each case $\mu=\mathrm{E} X_{1}$.

The rest of this paper consists of three sections: in Section 2 we prepare a series of important lemmas, in Section 3 we prove Theorem 1.1, and in Section 4 we propose two applications of Theorem 1.1 to risk theory and renewal theory.

\section{Lemmas}

Let the random variables $X_{k}, k=1, \ldots, n$, follow distributions $F_{k}, k=1, \ldots, n$, respectively, and let $C\left(u_{1}, \ldots, u_{n}\right):[0,1]^{n} \mapsto[0,1]$ be their copula, so that, by Sklar's theorem, it holds, for all $x_{k}, k=1, \ldots, n$, that

$$
\operatorname{Pr}\left(\bigcap_{k=1}^{n}\left(X_{k} \leq x_{k}\right)\right)=C\left(F_{1}\left(x_{1}\right), \ldots, F_{n}\left(x_{n}\right)\right) .
$$


In particular, if $F_{k}, k=1, \ldots, n$, are continuous then the copula $C\left(u_{1}, \ldots, u_{n}\right)$ fulfilling $(2.1)$ is unique and is identical to the joint distribution of the uniform variates $F\left(X_{k}\right), k=1, \ldots, n$. See Joe (1997) and Nelsen (2006) for comprehensive treatments on copulas.

For every nonempty subset $I$ of $\{1, \ldots, n\}$, the corresponding marginal copula is

$$
C\left(u_{i}: i \in I\right)=\left.C\left(u_{1}, \ldots, u_{n}\right)\right|_{u_{j}=1 \text { for } j \in\{1, \ldots, n\} \backslash I}
$$

and the corresponding marginal copula density, if it exists, is equal to

$$
c\left(u_{i}: i \in I\right)=\left(\prod_{i \in I} \frac{\partial}{\partial u_{i}}\right) C\left(u_{i}: i \in I\right) .
$$

Trivially, if the copula density $c\left(u_{1}, \ldots, u_{n}\right)$ is bounded over $\left(u_{1}, \ldots, u_{n}\right) \in[0,1]^{n}$, so is every marginal copula density.

Motivated by Corollary 3.1 of Ko and Tang (2008), in the following lemma we show a sufficient condition for the random variables $X_{k}, k=1, \ldots, n$, to be LEND or UEND.

Lemma 2.1. Assume that random variables $X_{k}, k=1, \ldots, n$, have continuous distributions $F_{k}, k=1, \ldots, n$, respectively, and possess a copula $C\left(u_{1}, \ldots, u_{n}\right)$ with a copula density $c\left(u_{1}, \ldots, u_{n}\right)$ well defined on $(0,1)^{n}$.

(a) If every marginal copula density is bounded in a neighbourhood of the origin (whose coordinates are all 0) then the $X_{k}, k=1, \ldots, n$, are LEND.

(b) If every marginal copula density is bounded in a neighbourhood of the ultimate vertex (whose coordinates are all 1 ) then the $X_{k}, k=1, \ldots, n$, are UEND.

Proof. We only prove (a) since (b) can be proven in the same way. Let $\varepsilon \in(0,1)$ and $M^{*}>0$ be two constants such that every marginal copula density is bounded by $M^{*}$ as long as all its arguments fall into the interval $(0, \varepsilon]$. Then, for arbitrarily given $x_{k}, k=1, \ldots, n$, denote by $I$ the set of all $i$ for which $F_{i}\left(x_{i}\right) \leq \varepsilon$ and by $I^{\mathrm{c}}$ its complement, $I^{\mathrm{c}}=\{1, \ldots, n\} \backslash I$. When $I$ is nonempty, we have

$$
\begin{aligned}
\operatorname{Pr}\left(\bigcap_{k=1}^{n}\left(X_{k} \leq x_{k}\right)\right) & \leq \operatorname{Pr}\left(\bigcap_{i \in I}\left(X_{i} \leq x_{i}\right)\right) \\
& =C\left(F_{i}\left(x_{i}\right): i \in I\right) \\
& =\int \cdots \int_{0<u_{i} \leq F_{i}\left(x_{i}\right), i \in I} c\left(u_{i}: i \in I\right) \prod_{i \in I} \mathrm{~d} u_{i} \\
& \leq M^{*} \prod_{i \in I} F_{i}\left(x_{i}\right) \\
& \leq M^{*} \prod_{i \in I} F_{i}\left(x_{i}\right) \prod_{j \in I^{c}} \frac{F_{j}\left(x_{j}\right)}{\varepsilon} \\
& \leq \frac{M^{*}}{\varepsilon^{n}} \prod_{k=1}^{n} F_{k}\left(x_{k}\right) .
\end{aligned}
$$

Clearly, if $I$ is empty then we have

$$
\operatorname{Pr}\left(\bigcap_{k=1}^{n}\left(X_{k} \leq x_{k}\right)\right) \leq 1 \leq \frac{1}{\varepsilon^{n}} \prod_{k=1}^{n} F_{k}\left(x_{k}\right) .
$$

Thus, inequality (1.1) holds with $M=\varepsilon^{-n}\left(M^{*} \vee 1\right)$. This completes the proof. 
Let us collect some preliminaries regarding the concept of END for later use. Throughout this paper, for a real number $x$, write $x^{+}=x \vee 0$ and $x^{-}=(-x) \vee 0$ as the positive and negative parts of $x$, respectively. The following lemma is essentially a refinement of Lemma 3.1 of Liu (2009).

Lemma 2.2. Let $X_{k}, k=1, \ldots, n$, be random variables, and let $g_{k}, k=1, \ldots, n$, be real functions.

(a) If the $X_{k}, k=1, \ldots, n$, are UEND with some dominating coefficient $M>0$ then

$$
\mathrm{E}\left(\prod_{k=1}^{n} X_{k}^{+}\right) \leq M \prod_{k=1}^{n} \mathrm{E} X_{k}^{+}
$$

(b) Assume that the $X_{k}, k=1, \ldots, n$, are LEND, UEND, or END with some dominating constant $M>0$. If the $g_{k}, k=1, \ldots, n$, are all nondecreasing then the $g_{k}\left(X_{k}\right), k=$ $1, \ldots, n$, are still LEND, UEND, or END, respectively, while if the $g_{k}, k=1, \ldots, n$, are all nonincreasing then the $g_{k}\left(X_{k}\right), k=1, \ldots, n$, are UEND, LEND, or END, respectively. For each case, the dominating constant $M>0$ remains unchanged.

Proof. (a) By Fubini's theorem and inequality (1.2), we have

$$
\begin{aligned}
\mathrm{E}\left(\prod_{k=1}^{n} X_{k}^{+}\right) & =\mathrm{E}\left(\int \cdots \int_{\left(x_{1}, \ldots, x_{n}\right) \in[0, \infty)^{n}}\left(\prod_{k=1}^{n} \mathbf{1}_{\left\{x_{k}<X_{k}^{+}\right\}}\right) \prod_{k=1}^{n} \mathrm{~d} x_{k}\right) \\
& =\int \cdots \int_{\left(x_{1}, \ldots, x_{n}\right) \in[0, \infty)^{n}} \operatorname{Pr}\left(\bigcap_{k=1}^{n}\left(X_{k}>x_{k}\right)\right) \prod_{k=1}^{n} \mathrm{~d} x_{k} \\
& \leq M \int \cdots \int_{\left(x_{1}, \ldots, x_{n}\right) \in[0, \infty)^{n}} \prod_{k=1}^{n} \operatorname{Pr}\left(X_{k}>x_{k}\right) \prod_{k=1}^{n} \mathrm{~d} x_{k} \\
& =M \prod_{k=1}^{n} \mathrm{E} X_{k}^{+} .
\end{aligned}
$$

Note that the derivation of (2.2) is still valid even when $\mathrm{E}_{k}^{+}=\infty$ for some $k=1, \ldots, n$.

(b) As all assertions can be proven in the same way, we only prove that if the $X_{k}, k=$ $1, \ldots, n$, are LEND with some dominating constant $M>0$ and the $g_{k}, k=1, \ldots, n$, are all nondecreasing, then the $g_{k}\left(X_{k}\right), k=1, \ldots, n$, are still LEND with the same dominating constant $M$. For each $k=1, \ldots, n$ and each real number $y_{k}$, the event $\left(g_{k}\left(X_{k}\right) \leq y_{k}\right)$ is equivalent to either $\Delta_{k}=\left(X_{k} \leq x_{k}\right)$ or $\Delta_{k}=\left(X_{k}<x_{k}\right)$ for $x_{k}=\sup \left\{x: g_{k}(x) \leq y_{k}\right\} \in$ $[-\infty, \infty]$. For the latter case, $\Delta_{k}=\left(X_{k}<x_{k}\right)$ can be approximated by $\left(X_{k} \leq x_{k}^{*}\right)$ as $x_{k}^{*} \rightarrow x_{k}-$. Therefore, by relation (1.1) and the continuity of the probability measure, we have

$$
\operatorname{Pr}\left(\bigcap_{k=1}^{n}\left(g_{k}\left(X_{k}\right) \leq y_{k}\right)\right)=\operatorname{Pr}\left(\bigcap_{k=1}^{n} \Delta_{k}\right) \leq M \prod_{k=1}^{n} \operatorname{Pr}\left(\Delta_{k}\right)=M \prod_{k=1}^{n} \operatorname{Pr}\left(g_{k}\left(X_{k}\right) \leq y_{k}\right) .
$$

This completes the proof.

The following generalized Borel-Cantelli lemma is due to Kochen and Stone (1964) and was retrieved recently in Yan (2006). 
Lemma 2.3. Let $\left\{A_{n}, n=1,2, \ldots\right\}$ be a sequence of events such that $\sum_{n=1}^{\infty} \operatorname{Pr}\left(A_{n}\right)=\infty$. Then

$$
\operatorname{Pr}\left(A_{n} \text { infinitely often }\right) \geq \limsup _{n \rightarrow \infty} \frac{\sum_{1 \leq i<j \leq n} \operatorname{Pr}\left(A_{i}\right) \operatorname{Pr}\left(A_{j}\right)}{\sum_{1 \leq i<j \leq n} \operatorname{Pr}\left(A_{i} A_{j}\right)} .
$$

Let $F$ be a distribution on $(-\infty, \infty)$. For arbitrarily fixed $\delta>0$, define auxiliary functions $f_{\delta}$ and $f_{\delta}^{ \pm}$as

$$
\begin{aligned}
f_{\delta}(x) & =x^{-\delta} \int_{|y| \leq x}|y|^{1+\delta} F(\mathrm{~d} y) \\
& =x^{-\delta} \int_{0 \leq y \leq x} y^{1+\delta} F(\mathrm{~d} y)+x^{-\delta} \int_{-x \leq y \leq 0}(-y)^{1+\delta} F(\mathrm{~d} y) \\
& =f_{\delta}^{+}(x)+f_{\delta}^{-}(x), \quad x>0 .
\end{aligned}
$$

These auxiliary functions will be crucial for establishing our key inequalities for the tail probabilities of the sums of END random variables. The following result is elementary.

Lemma 2.4. For the auxiliary functions $f_{\delta}$ and $f_{\delta}^{ \pm}$defined in (2.3), as $x \rightarrow \infty$,

(a) if $x \operatorname{Pr}(X>x) \rightarrow 0$ then $f_{\delta}^{+}(x) \rightarrow 0$;

(b) if $x \operatorname{Pr}(X<-x) \rightarrow 0$ then $f_{\delta}^{-}(x) \rightarrow 0$;

(c) if $x \operatorname{Pr}(|X|>x) \rightarrow 0$ then $f_{\delta}(x)=f_{\delta}^{+}(x)+f_{\delta}^{-}(x) \rightarrow 0$.

Proof. We only prove (a) since (b) can be proven in the same way and (c) is an immediate consequence of (a) and (b). By Fubini's theorem,

$$
\begin{aligned}
f_{\delta}^{+}(x) & =\frac{1+\delta}{x^{\delta}} \int_{0}^{x}\left(\int_{0}^{y} z^{\delta} \mathrm{d} z\right) F(\mathrm{~d} y) \\
& =\frac{1+\delta}{x^{\delta}} \int_{0}^{x} \int_{z}^{x} z^{\delta} F(\mathrm{~d} y) \mathrm{d} z \\
& \leq \frac{1+\delta}{x^{\delta}} \int_{0}^{x} z^{\delta} \bar{F}(z) \mathrm{d} z .
\end{aligned}
$$

For every $\varepsilon>0$, there is some $z_{0}>0$ such that $\bar{F}(z) \leq \varepsilon z^{-1}$ for all $z>z_{0}$. Thus,

$$
\begin{aligned}
f_{\delta}^{+}(x) & \leq \frac{1+\delta}{x^{\delta}}\left(\int_{0}^{z_{0}} z^{\delta} \bar{F}(z) \mathrm{d} z+\varepsilon \int_{z_{0}}^{x} z^{\delta-1} \mathrm{~d} z\right) \\
& \leq \frac{1+\delta}{x^{\delta}}\left(\int_{0}^{z_{0}} z^{\delta} \bar{F}(z) \mathrm{d} z+\frac{\varepsilon}{\delta} x^{\delta}\right) .
\end{aligned}
$$

By the arbitrariness of $\varepsilon$, we conclude that $f_{\delta}^{+}(x) \rightarrow 0$ as $x \rightarrow \infty$. This completes the proof.

Let $\left\{X_{k}, k=1,2, \ldots\right\}$ be a sequence of UEND random variables with common distribution $F$ and mean 0 . For arbitrarily fixed $0<v<1$, define

$$
\tilde{X}_{k}=-v x \mathbf{1}_{\left\{X_{k}<-v x\right\}}+X_{k} \mathbf{1}_{\left\{-v x \leq X_{k} \leq v x\right\}}+v x \mathbf{1}_{\left\{X_{k}>v x\right\}}, \quad k=1,2, \ldots,
$$

which, by Lemma 2.2(b), are still UEND random variables. Write

$$
\tilde{S}_{n}=\sum_{k=1}^{n} \tilde{X}_{k}, \quad n=1,2, \ldots,
$$

and $\mu_{ \pm}=\mathrm{E} X_{1}^{ \pm}$. Trivially, $\mu_{+}=\mu_{-}$since $\mathrm{E} X_{1}=0$. 
We are going to establish some key inequalities of large deviation type for the sums $\tilde{S}_{n}, n=$ $1,2, \ldots$ An important feature of the following result is that it does not require $X_{1}$ to have a finite moment of order higher than 1 . The obtained inequality is new even for the independent case.

Lemma 2.5. Consider the truncated random variables defined in (2.4), where the $X_{k}, k=$ $1,2, \ldots$, are UEND random variables with common distribution $F$, mean 0 , and a dominating constant $M>0$. Then, for every $v>0, \gamma>0,0<\delta \leq 1$, and $0<\theta<1$, there is some $x_{0}=x_{0}(v, \gamma, \delta, \theta)>0$ such that, for all $n=1,2, \ldots$ and $x \geq(\gamma n) \vee x_{0}$,

$$
\operatorname{Pr}\left(\tilde{S}_{n}>x\right) \leq M\left(f_{\delta}^{+}(v x)+v x \bar{F}(v x)\right)^{(1-\theta) / v},
$$

where the auxiliary function $f_{\delta}^{+}$is defined in (2.3).

Proof. By Lemma 2.2(b), for every $v>0$ and $h>0$, the random variables $h \tilde{X}_{k}, k=$ $1,2, \ldots$, are still UEND with the same dominating constant $M$ unrelated to $v$ or $h$. Let $h=h(x)$ be a positive function of $x$ whose exact form will be specified later such that $h(x) \rightarrow 0$ as $x \rightarrow \infty$. By Markov's inequality and Lemma 2.2(a), we have

$$
\operatorname{Pr}\left(\tilde{S}_{n}>x\right) \leq \mathrm{e}^{-h x} \mathrm{Ee}^{h \tilde{S}_{n}} \leq M \mathrm{e}^{-h x}\left(\mathrm{E} \mathrm{e}^{h \tilde{X}_{1}}\right)^{n} .
$$

Now we focus on the estimation of the exponential moment $\mathrm{E}^{h \tilde{X}_{1}}$. Clearly,

$$
\begin{aligned}
\mathrm{Ee}^{h \tilde{X}_{1}}= & \left(\int_{-v x \leq y \leq 0}+\int_{0 \leq y \leq v x}\right)\left(\mathrm{e}^{h y}-1\right) F(\mathrm{~d} y)+\left(\mathrm{e}^{h v x}-1\right) \bar{F}(v x) \\
& +\left(\mathrm{e}^{-h v x}-1\right) F(-v x-0)+1 \\
\leq & \left(\int_{-v x \leq y \leq 0}+\int_{0 \leq y \leq v x}\right)\left(\mathrm{e}^{h y}-1\right) F(\mathrm{~d} y)+\left(\mathrm{e}^{h v x}-1\right) \bar{F}(v x)+1 \\
= & I_{1}(x)+I_{2}(x)+I_{3}(x)+1 .
\end{aligned}
$$

We use an idea of Tang and Yan (2002) to deal with $I_{1}(x)$. It holds for $y \leq 0$ that

$$
0 \leq \frac{\mathrm{e}^{h y}-1-h y}{h} \leq y\left(\mathrm{e}^{h y}-1\right) \leq-y .
$$

Then, by the dominated convergence theorem,

$$
\lim _{x \rightarrow \infty} \frac{I_{1}(x)}{h}=\int_{-\infty}^{0} \lim _{h \rightarrow 0+} \frac{\mathrm{e}^{h y}-1-h y}{h} F(\mathrm{~d} y)-\mu_{-}=-\mu_{-} .
$$

This means that

$$
I_{1}(x)=-\mu_{-} h+o_{1}(h),
$$

where $o_{1}(h)$ is a real function of $h>0$ satisfying $o_{1}(h) / h \rightarrow 0$ as $h \rightarrow 0+$. For arbitrarily fixed $0<\delta \leq 1$, by the monotonicity of the function $\left(\mathrm{e}^{h y}-1-h y\right) / y^{1+\delta}$ for $y>0$, we have

$$
\begin{aligned}
I_{2}(x) & \leq \int_{0}^{v x} \frac{\mathrm{e}^{h y}-1-h y}{y^{1+\delta}} y^{1+\delta} F(\mathrm{~d} y)+\mu_{+} h \\
& \leq \frac{\mathrm{e}^{h v x}-1}{(v x)^{1+\delta}} \int_{0}^{v x} y^{1+\delta} F(\mathrm{~d} y)+\mu_{+} h \\
& =\frac{\mathrm{e}^{h v x}-1}{h v x} f_{\delta}^{+}(v x) h+\mu_{+} h .
\end{aligned}
$$


Rewrite $I_{3}(x)$ as

We specify $h$ to be

$$
I_{3}(x)=\frac{\mathrm{e}^{h v x}-1}{h v x}(v x \bar{F}(v x)) h
$$

$$
h=\frac{1}{v x} \ln \left(1+\frac{1}{f_{\delta}^{+}(v x)+v x \bar{F}(v x)}\right) .
$$

Note that $h \rightarrow 0+$ is equivalent to $x \rightarrow \infty$. Actually, the fact that $h \rightarrow 0+$ implies that $x \rightarrow \infty$ is trivial since both $f_{\delta}^{+}(v x)$ and $v x \bar{F}(v x)$ are bounded for $x>0$. For the other implication, with some $x^{*}>0$ such that $F\left(v x^{*}\right)-F(0)>0$, it holds for all $x \geq x^{*}$ that

$$
h \leq \frac{1}{v x} \ln \left(1+\frac{1}{f_{\delta}^{+}(v x)}\right) \leq \frac{1}{v x} \ln \left(1+\frac{(v x)^{\delta}}{\int_{0}^{v x^{*}} y^{1+\delta} F(\mathrm{~d} y)}\right) \sim \frac{\delta \ln x}{v x} .
$$

Substituting (2.8)-(2.11) into (2.7) and noting that both $f_{\delta}^{+}(v x)$ and $v x \bar{F}(v x)$ converge to 0 as $x \rightarrow \infty$, we obtain

$$
\begin{aligned}
\mathrm{Ee}^{h \tilde{X}_{k}} & \leq o_{1}(h)+\frac{\mathrm{e}^{h v x}-1}{h v x}\left(f_{\delta}^{+}(v x)+v x \bar{F}(v x)\right) h+1 \\
& =o_{1}(h)+\frac{h}{\ln \left(1+1 /\left(f_{\delta}^{+}(v x)+v x \bar{F}(v x)\right)\right)}+1 \\
& =o_{2}(h)+1,
\end{aligned}
$$

where $o_{2}(h)$ is a real function of $x>0$ satisfying $o_{2}(h) / h \rightarrow 0$ as $x \rightarrow \infty$. Substituting (2.12) into (2.6) and using the elementary inequality $1+z \leq \mathrm{e}^{z}$ for every real number $z$, we have, for all $n=1,2, \ldots$ and $x \geq \gamma n$,

$$
\operatorname{Pr}\left(\tilde{S}_{n}>x\right) \leq M \exp \left\{o_{2}(h) n-h x\right\} \leq M \exp \left\{\left(\frac{\left|o_{2}(h)\right|}{\gamma h}-1\right) h x\right\} .
$$

For arbitrarily fixed $0<\theta<1$, there is some large $x_{0}>0$ such that, for all $x \geq x_{0}$,

$$
\frac{\left|o_{2}(h)\right|}{\gamma h} \leq \theta
$$

It follows that, for all $n=1,2, \ldots$ and $x \geq(\gamma n) \vee x_{0}$,

$$
\operatorname{Pr}\left(\tilde{S}_{n}>x\right) \leq M \exp \{-(1-\theta) h x\}=M\left(1+\frac{1}{f_{\delta}^{+}(v x)+v x \bar{F}(v x)}\right)^{-(1-\theta) / v},
$$

yielding inequality (2.5). This completes the proof.

By a symmetrization procedure, it is easy to apply Lemma 2.5 to establish a similar inequality for the tail probabilities of $\left|\tilde{S}_{n}\right|, n=1,2, \ldots$.

Lemma 2.6. Consider the truncated random variables defined in (2.4), where the $X_{k}, k=$ $1,2, \ldots$, are END random variables with common distribution $F$, mean 0 , and a dominating constant $M>0$. Then, for every $v>0, \gamma>0,0<\delta \leq 1$, and $0<\theta<1$, there is some $x_{0}=x_{0}(v, \gamma, \delta, \theta)>0$ such that, for all $n=1,2, \ldots$ and $x \geq(\gamma n) \vee x_{0}$,

$$
\operatorname{Pr}\left(\left|\tilde{S}_{n}\right|>x\right) \leq 2 M\left(f_{\delta}(v x)+v x \operatorname{Pr}\left(\left|X_{1}\right|>v x\right)\right)^{(1-\theta) / v},
$$

where the auxiliary function $f_{\delta}$ is defined in (2.3). 
Proof. Recall the other two auxiliary functions $f_{\delta}^{ \pm}$defined in (2.3). Note that, by Lemma 2.2(b), if the random variables $X_{k}, k=1,2, \ldots$, are LEND then the $-\tilde{X}_{k}, k=1,2, \ldots$, are UEND. Therefore, applying Lemma 2.5 to the random variables $-\tilde{X}_{k}, k=1,2, \ldots$, there is some $x_{0}=x_{0}(v, \gamma, \delta, \theta)>0$ such that, for all $n=1,2, \ldots$ and $x \geq(\gamma n) \vee x_{0}$,

$$
\operatorname{Pr}\left(-\tilde{S}_{n}>x\right) \leq M\left(f_{\delta}^{-}(v x)+v x \operatorname{Pr}\left(-X_{1}>v x\right)\right)^{(1-\theta) / v} .
$$

A simple combination of inequalities (2.5) and (2.13) yields, for a suitably modified constant $x_{0}=x_{0}(v, \gamma, \delta, \theta)>0$ and all $n=1,2, \ldots, x \geq(\gamma n) \vee x_{0}$,

$$
\begin{aligned}
\operatorname{Pr}\left(\left|\tilde{S}_{n}\right|>x\right) \leq & M\left(f_{\delta}^{+}(v x)+v x \operatorname{Pr}\left(X_{1}>v x\right)\right)^{(1-\theta) / v} \\
& +M\left(f_{\delta}^{-}(v x)+v x \operatorname{Pr}\left(X_{1}<-v x\right)\right)^{(1-\theta) / v} \\
\leq & 2 M\left(f_{\delta}(v x)+v x \operatorname{Pr}\left(\left|X_{1}\right|>v x\right)\right)^{(1-\theta) / v} .
\end{aligned}
$$

This proves the assertion of Lemma 2.6.

The following result essentially shows that, once $X_{1}$ has a finite moment of order higher than 1 , by suitably choosing the values of $\theta$ and $v$ in Lemma 2.6, the tail probability $\operatorname{Pr}\left(\left|\tilde{S}_{n}\right|>x\right)$ can decay to 0 faster than any power rate. Such a result is of independent interest, particularly in the study of large deviations. Lemma 2.3 of Tang (2006) gives a result of the same flavour for negatively dependent random variables.

Corollary 2.1. Recall the truncated random variables defined in (2.4).

(a) In addition to the conditions of Lemma 2.5, assume that $\mathrm{E}\left(X_{1}^{+}\right)^{1+\delta}<\infty$ for some $0<\delta \leq 1$. Then, for every $v>0, \gamma>0$, and $0<\theta<1$, there is some $K=K(v, \gamma, \delta, \theta)>0$ such that, for all $n=1,2, \ldots$ and $x \geq \gamma n$,

$$
\operatorname{Pr}\left(\tilde{S}_{n}>x\right) \leq K x^{-\delta(1-\theta) / v} .
$$

(b) In addition to the conditions of Lemma 2.6, assume that $\mathrm{E}\left|X_{1}\right|^{1+\delta}<\infty$ for some $0<\delta \leq 1$. Then, for every $v>0, \gamma>0$, and $0<\theta<1$, there is some $K=K(v, \gamma, \delta, \theta)>0$ such that, for all $n=1,2, \ldots$ and $x \geq \gamma n$,

$$
\operatorname{Pr}\left(\left|\tilde{S}_{n}\right|>x\right) \leq K x^{-\delta(1-\theta) / v} .
$$

Proof. (a) Lemma 2.5 asserts that there is some $x_{0}=x_{0}(v, \gamma, \delta, \theta)>0$ such that inequality (2.5) holds for all $n=1,2, \ldots$ and $x \geq(\gamma n) \vee x_{0}$. Using Markov's inequality on the right-hand side of (2.5), it holds for all $n=1,2, \ldots$ and $x \geq(\gamma n) \vee x_{0}$ that

$$
\begin{aligned}
\operatorname{Pr}\left(\tilde{S}_{n}>x\right) & \leq M\left((v x)^{-\delta} \int_{0}^{v x} y^{1+\delta} F(\mathrm{~d} y)+v x \frac{1}{(v x)^{1+\delta}} \mathrm{E}\left(X_{1}^{+}\right)^{1+\delta}\right)^{(1-\theta) / v} \\
& \leq M\left(2 v^{-\delta} \mathrm{E}\left(X_{1}^{+}\right)^{1+\delta}\right)^{(1-\theta) / v} x^{-\delta(1-\theta) / v} .
\end{aligned}
$$

Furthermore, it trivially holds for all $n=1,2, \ldots$ and $\gamma n \leq x \leq x_{0}$ that

$$
\operatorname{Pr}\left(\tilde{S}_{n}>x\right) \leq 1 \leq x_{0}^{\delta(1-\theta) / v} x^{-\delta(1-\theta) / v} .
$$

Therefore, inequality (2.14) holds with

$$
K=M\left(2 v^{-\delta} \mathrm{E}\left(X_{1}^{+}\right)^{1+\delta}\right)^{(1-\theta) / v} \vee x_{0}^{\delta(1-\theta) / v}
$$

for all $n=1,2, \ldots$ and $x \geq \gamma n$. 
(b) Applying Corollary 2.1(a) to the random variables $-\tilde{X}_{k}, k=1,2, \ldots$, there is some $K=K(v, \gamma, \delta, \theta)>0$ such that, for all $n=1,2, \ldots$ and $x \geq \gamma n$,

$$
\operatorname{Pr}\left(-\tilde{S}_{n}>x\right) \leq K x^{-\delta(1-\theta) / v} .
$$

A simple combination of inequalities (2.14) and (2.16) yields relation (2.15) with a suitably modified constant $K>0$. This completes the proof.

\section{Proof of Theorem 1.1}

\subsection{Proof of the necessity part}

Assume that $S_{n} / n \stackrel{\text { a.s. }}{\rightarrow} \mu$ as $n \rightarrow \infty$. This condition implies that $X_{n} / n \stackrel{\text { a.s. }}{\rightarrow} 0$ as $n \rightarrow \infty$, and, hence, that both $X_{n}^{+} / n \stackrel{\text { a.s. }}{\rightarrow} 0$ and $X_{n}^{-} / n \stackrel{\text { a.s. }}{\rightarrow} 0$ as $n \rightarrow \infty$. Define $A_{n}=\left(X_{n}^{+}>n\right)$ for $n=1,2, \ldots$. We have $\operatorname{Pr}\left(A_{n}\right.$ infinitely often $)=0$. This implies that

$$
\sum_{n=1}^{\infty} \operatorname{Pr}\left(A_{n}\right)<\infty
$$

because otherwise, by Lemma 2.3 and the pairwise UEND of $\left\{X_{k}, k=1,2, \ldots\right\}$, we would have

$$
\operatorname{Pr}\left(A_{n} \text { infinitely often }\right) \geq \limsup _{n \rightarrow \infty} \frac{\sum_{1 \leq i<j \leq n} \operatorname{Pr}\left(A_{i}\right) \operatorname{Pr}\left(A_{j}\right)}{\sum_{1 \leq i<j \leq n} \operatorname{Pr}\left(A_{i} A_{j}\right)} \geq \frac{1}{M} .
$$

Hence,

$$
\mathrm{E} X_{1}^{+}=\sum_{n=0}^{\infty} \int_{n}^{n+1} \operatorname{Pr}\left(X_{1}>x\right) \mathrm{d} x \leq 1+\sum_{n=1}^{\infty} \operatorname{Pr}\left(A_{n}\right)<\infty .
$$

In the same way, we can prove that $\mathrm{E} X_{1}^{-}<\infty$. Therefore, $\mathrm{E}\left|X_{1}\right|<\infty$. Finally, by the sufficiency part of Theorem 1.1 which we are to prove below, the finiteness of $\mathrm{E}\left|X_{1}\right|$ implies that $S_{n} / n \stackrel{\text { a.s. }}{\rightarrow}$ E $X_{1}$ as $n \rightarrow \infty$. Hence, $\mu=\mathrm{E} X_{1}$.

\subsection{Proof of the sufficiency part}

Write $S_{n}^{( \pm)}=\sum_{k=1}^{n} X_{k}^{ \pm}$for $n=1,2, \ldots$ and write $\mu_{ \pm}=\mathrm{E} X_{1}^{ \pm}$as before. Clearly, it suffices to prove that both $S_{n}^{(+)} / n \stackrel{\text { a.s. }}{\rightarrow} \mu_{+}$and $S_{n}^{(-)} / n \stackrel{\text { a.s. }}{\rightarrow} \mu_{-}$hold as $n \rightarrow \infty$. We prove only the former since the latter can be proven in the same way.

For arbitrarily fixed $v>0$ and $n=1,2, \ldots$, similarly as in (2.4) we define

$$
\tilde{X}_{k, n}^{+}=-v n \mathbf{1}_{\left\{X_{k}^{+}-\mu_{+}<-v n\right\}}+\left(X_{k}^{+}-\mu_{+}\right) \mathbf{1}_{\left\{-v n \leq X_{k}^{+}-\mu_{+} \leq v n\right\}}+v n \mathbf{1}_{\left\{X_{k}^{+}-\mu_{+}>v n\right\}}
$$

for $k=1, \ldots, n$. Write $\tilde{S}_{n}^{(+)}=\sum_{k=1}^{n} \tilde{X}_{k, n}^{+}$. Let $\varepsilon>0$ and $\alpha>1$ be arbitrarily fixed and, as usual, denote by $[z]$ the largest integer that is not larger than $z$. By Lemma 2.6, with suitably chosen $0<v<1$ and $0<\theta<1$ such that $(1-\theta) / v=1$, there is some positive integer $n_{0}=n_{0}(v, \varepsilon, \delta, \theta)$ such that, for all $n \geq n_{0}$,

$$
\operatorname{Pr}\left(\left|\frac{\tilde{S}_{n}^{(+)}}{n}\right|>\varepsilon\right) \leq 2 M\left((v \varepsilon n)^{-\delta} \int_{|y| \leq v \varepsilon n}|y|^{1+\delta} \tilde{F}^{+}(\mathrm{d} y)+v \varepsilon n \operatorname{Pr}\left(\left|X_{1}^{+}-\mu_{+}\right|>v \varepsilon n\right)\right),
$$

where $\tilde{F}^{+}$denotes the distribution of $X_{1}^{+}-\mu_{+}$. It follows that

$$
\sum_{n=1}^{\infty} \operatorname{Pr}\left(\left|\frac{\tilde{S}_{\left[\alpha^{n}\right]}^{(+)}}{\left[\alpha^{n}\right]}\right|>\varepsilon\right) \leq \log _{\alpha} n_{0}+2 M\left(v \varepsilon \alpha^{-1}\right)^{-\delta} \Sigma_{1}+2 M v \varepsilon \Sigma_{2}
$$


with

$$
\Sigma_{1}=\sum_{n=1}^{\infty} \alpha^{-\delta n} \int_{|y| \leq v \varepsilon \alpha^{n}}|y|^{1+\delta} \tilde{F}^{+}(\mathrm{d} y), \quad \Sigma_{2}=\sum_{n=1}^{\infty}\left[\alpha^{n}\right] \operatorname{Pr}\left(\left|X_{1}^{+}-\mu_{+}\right|>v \varepsilon\left[\alpha^{n}\right]\right) .
$$

Interchanging the order of the sum and integral in $\Sigma_{1}$, we see that, for some constant $K_{1}>0$,

$$
\begin{aligned}
\Sigma_{1} & =\int_{-\infty}^{\infty}\left(\sum_{n \geq\left(\log _{\alpha}|y|-\log _{\alpha} v \varepsilon\right) \vee 1} \alpha^{-\delta n}\right)|y|^{1+\delta} \tilde{F}^{+}(\mathrm{d} y) \\
& =\int_{-\infty}^{\infty} \frac{\alpha^{-\delta\left(\left(\log _{\alpha}|y|-\log _{\alpha} v \varepsilon\right) \vee 1\right)}}{1-\alpha^{-\delta}}|y|^{1+\delta} \tilde{F}^{+}(\mathrm{d} y) \\
& \leq K_{1} \int_{-\infty}^{\infty}|y| \tilde{F}^{+}(\mathrm{d} y) \\
& =K_{1} \mathrm{E}\left|X_{1}^{+}-\mu_{+}\right| \\
& <\infty
\end{aligned}
$$

Moreover, it holds for some constant $K_{2}>0$ that

$$
\begin{aligned}
\Sigma_{2} & \leq \sum_{n=1}^{\infty} \frac{\left[\alpha^{n}\right]}{\left[(\alpha-1) \alpha^{n-1}\right]} \sum_{k=1}^{\left[(\alpha-1) \alpha^{n-1}\right]} \operatorname{Pr}\left(\left|X_{1}^{+}-\mu_{+}\right|>v \varepsilon\left(\left[\alpha^{n-1}\right]+k\right)\right) \\
& \leq K_{2} \sum_{n=1}^{\infty} \operatorname{Pr}\left(\left|X_{1}^{+}-\mu_{+}\right|\right)>v \varepsilon n \\
& \leq \frac{K_{2}}{v \varepsilon} \mathrm{E}\left|X_{1}^{+}-\mu_{+}\right| \\
& <\infty
\end{aligned}
$$

where the summation $\sum_{k=1}^{\left[(\alpha-1) \alpha^{n-1}\right]}$ produces a value 0 in the case where $\left[(\alpha-1) \alpha^{n-1}\right]=0$. Substituting (3.2) and (3.3) into (3.1) yields

$$
\sum_{n=1}^{\infty} \operatorname{Pr}\left(\left|\frac{\tilde{S}_{\left[\alpha^{n}\right]}^{(+)}}{\left[\alpha^{n}\right]}\right|>\varepsilon\right)<\infty
$$

Hence,

$$
\begin{aligned}
& \operatorname{Pr}\left(\left|\frac{S_{\left[\alpha^{n}\right]}^{(+)}}{\left[\alpha^{n}\right]}-\mu_{+}\right|>\varepsilon \text { infinitely often }\right) \\
& \quad \leq \operatorname{Pr}\left(\left|\frac{\tilde{S}_{\left[\alpha^{n}\right]}^{(+)}}{\left[\alpha^{n}\right]}\right|>\varepsilon \text { infinitely often }\right)+\operatorname{Pr}\left(\frac{S_{\left[\alpha^{n}\right]}^{(+)}}{\left[\alpha^{n}\right]}-\mu_{+} \neq \frac{\tilde{S}_{\left[\alpha^{n}\right]}^{(+)}}{\left[\alpha^{n}\right]} \text { infinitely often }\right) \\
& \quad \leq \lim _{m \rightarrow \infty} \operatorname{Pr}\left(\bigcup_{n=m}^{\infty}\left(\frac{\tilde{S}_{\left[\alpha^{n}\right]}^{(+)}}{\left[\alpha^{n}\right]}>\varepsilon\right)\right)+\lim _{m \rightarrow \infty} \operatorname{Pr}\left(\bigcup_{n=m}^{\infty} \bigcup_{k=1}^{\left[\alpha^{n}\right]}\left(X_{k}^{+}-\mu_{+} \neq \tilde{X}_{k,\left[\alpha^{n}\right]}^{+}\right)\right) \\
& \quad \leq \limsup _{m \rightarrow \infty} \sum_{n=m}^{\infty} \operatorname{Pr}\left(\frac{\tilde{S}_{\left[\alpha^{n}\right]}^{(+)}}{\left[\alpha^{n}\right]}>\varepsilon\right)+\limsup _{m \rightarrow \infty} \sum_{n=m}^{\infty}\left[\alpha^{n}\right] \operatorname{Pr}\left(X_{k}-\mu_{+}>v\left[\alpha^{n}\right]\right) \\
& \quad=0,
\end{aligned}
$$

where in the last step the convergence of the last series can be verified in the same way as (3.3). 
This proves that

$$
\lim _{n \rightarrow \infty} \frac{S_{\left[\alpha^{n}\right]}^{(+)}}{\left[\alpha^{n}\right]} \stackrel{\text { a.s. }}{=} \mu_{+}
$$

For every positive integer $n$, there is a unique positive integer $k_{n}$ such that $\left[\alpha^{k_{n}-1}\right] \leq n<\left[\alpha^{k_{n}}\right]$. Hence,

$$
\frac{\left[\alpha^{k_{n}-1}\right]}{\left[\alpha^{k_{n}}\right]} \frac{S_{\left[\alpha^{k_{n}-1}\right]}^{(+)}}{\left[\alpha^{k_{n}-1}\right]}=\frac{S_{\left[\alpha^{k_{n}-1}\right]}^{(+)}}{\left[\alpha^{k_{n}}\right]} \leq \frac{S_{n}^{(+)}}{n} \leq \frac{S_{\left[\alpha^{k_{n}}\right]}^{(+)}}{\left[\alpha^{k_{n}-1}\right]}=\frac{\left[\alpha^{k_{n}}\right]}{\left[\alpha^{k_{n}-1}\right]} \frac{S_{\left[\alpha^{k_{n}}\right]}^{(+)}}{\left[\alpha^{k_{n}}\right]} .
$$

It follows from (3.4) and (3.5) that

$$
\frac{\mu_{+}}{\alpha} \stackrel{\text { a.s. }}{\leq} \liminf _{n \rightarrow \infty} \frac{S_{n}^{(+)}}{n} \leq \limsup _{n \rightarrow \infty} \frac{S_{n}^{(+)}}{n} \stackrel{\text { a.s. }}{\leq} \alpha \mu_{+} .
$$

By the arbitrariness of $\alpha$ we obtain $S_{n}^{(+)} / n \stackrel{\text { a.s. }}{\rightarrow} \mu_{+}$as $n \rightarrow \infty$.

\section{Applications}

\subsection{Application to risk theory}

Let $\left\{X_{k}, k=1,2, \ldots\right\}$ be a sequence of random variables with partial sums $S_{n}, n=$ $1,2, \ldots$, and let $N$ be a nonnegative integer-valued random variable independent of $\left\{X_{k}, k=\right.$ $1,2, \ldots\}$. The study of the tail behaviour of the random sum

$$
S_{N}=\sum_{k=1}^{N} X_{k}
$$

is of fundamental interest in various areas of applied probability. Robert and Segers (2008) interpreted $S_{N}$ as the total amount of claims of an insurance portfolio in earthquake insurance. Assuming that $N$ has a consistently varying tail and that the $X_{k}, k=1,2, \ldots$, are independent, identically distributed, and nonnegative, with tails relatively lighter than that of $N$, they showed that the tail behaviour of $S_{N}$ is mainly determined by that of $N$. See also Aleškevičiene et al. (2008) and Denisov et al. (2010) for some extensions.

With the help of Theorem 1.1 and Corollary 2.1, we are able to relax the independence assumption on $\left\{X_{k}, k=1,2, \ldots\right\}$ to END. This is particularly relevant in insurance, in view of the fact that claims from an insurance portfolio, or within a given reference period, are generated in the same or similar situations and, hence, they should be dependent.

A distribution $G$ on $[0, \infty)$ is said to be of consistent variation, written as $G \in \mathcal{C}$, if

$$
\lim _{y \rightarrow 1-} \limsup _{x \rightarrow \infty} \frac{\bar{G}(x y)}{\bar{G}(x)}=1, \quad \text { or, equivalently, } \quad \lim _{y \rightarrow 1+} \liminf _{x \rightarrow \infty} \frac{\bar{G}(x y)}{\bar{G}(x)}=1 .
$$

Note that the class $\mathcal{C}$ contains all distributions of regular variation. Clearly, if $G \in \mathcal{C}$ then, necessarily, $\bar{G}(x y)=O(\bar{G}(x))$ for every $y>0$. Furthermore, by Lemma 3.5 of Tang and Tsitsiashvili (2003), there is some constant $p>0$ such that

$$
x^{-p}=o(\bar{G}(x)) .
$$


Theorem 4.1. Consider the random sum (4.1) in which $\left\{X_{k}, k=1,2, \ldots\right\}$ is a sequence of END random variables with common distribution $F$, mean $\mu>0$, and $\mathrm{E}\left|X_{1}\right|^{1+\delta}<\infty$ for some $\delta>0$, while $N$ follows a distribution $G \in \mathcal{C}$. As $x \rightarrow \infty$, the relation

$$
\operatorname{Pr}\left(S_{N}>x\right) \sim \bar{G}\left(\frac{x}{\mu}\right)
$$

holds under one of the following groups of conditions:

(a) $x \operatorname{Pr}\left(\left|X_{1}\right|>x\right)=o(\bar{G}(x))$;

(b) $\mathrm{E} N<\infty$ and $\operatorname{Pr}\left(\left|X_{1}\right|>x\right)=o(\bar{G}(x))$.

Proof. For arbitrarily fixed $0<\varepsilon<1$, it is easy to see that

$$
\begin{aligned}
\operatorname{Pr}\left(S_{N}>x\right) & \leq \sum_{1 \leq n \leq(1-\varepsilon) x / \mu} \operatorname{Pr}\left(S_{n}>x\right) \operatorname{Pr}(N=n)+\operatorname{Pr}\left(N>\frac{(1-\varepsilon) x}{\mu}\right) \\
& =I(x)+\operatorname{Pr}\left(N>\frac{(1-\varepsilon) x}{\mu}\right) .
\end{aligned}
$$

Similarly as in (2.4), for arbitrarily fixed $v>0$, define

$$
\tilde{X}_{k}=-v x \mathbf{1}_{\left\{X_{k}-\mu<-v x\right\}}+\left(X_{k}-\mu\right) \mathbf{1}_{\left\{-v x \leq X_{k}-\mu \leq v x\right\}}+v x \mathbf{1}_{\left\{X_{k}-\mu>v x\right\}}, \quad k=1,2, \ldots
$$

Thus,

$$
\begin{aligned}
I(x) & \leq \sum_{1 \leq n \leq(1-\varepsilon) x / \mu} \operatorname{Pr}\left(S_{n}-n \mu>\varepsilon x\right) \operatorname{Pr}(N=n) \\
& \leq \sum_{1 \leq n \leq(1-\varepsilon) x / \mu}\left(n \operatorname{Pr}\left(\left|X_{1}-\mu\right|>v x\right)+\operatorname{Pr}\left(\sum_{k=1}^{n} \tilde{X}_{k}>\varepsilon x\right)\right) \operatorname{Pr}(N=n) .
\end{aligned}
$$

For the second term above, by Corollary 2.1(a), for $v, \varepsilon$, and $\delta$ given above and arbitrarily fixed $0<\theta<1$, there is some $K=K(v, \varepsilon, \delta, \theta)>0$ such that, for all $n=1,2, \ldots$,

$$
\operatorname{Pr}\left(\sum_{k=1}^{n} \tilde{X}_{k}>\varepsilon x\right) \leq K x^{-\delta(1-\theta) / v}
$$

Recall relation (4.2). We may suitably adjust the values of $v$ and $\theta$ such that $\delta(1-\theta) / v>p$. Substituting (4.6) into (4.5), under either condition (a) or condition (b), we have

$$
I(x) \leq \operatorname{Pr}\left(\left|X_{1}-\mu\right|>v x\right) \text { E } N \mathbf{1}_{\{N \leq(1-\varepsilon) x / \mu\}}+K x^{-p}=o(\bar{G}(x)) .
$$

It follows from this and (4.4) that

$$
\limsup _{x \rightarrow \infty} \frac{\operatorname{Pr}\left(S_{N}>x\right)}{\operatorname{Pr}(N>x / \mu)} \leq \lim _{\varepsilon \rightarrow 0+} \limsup _{x \rightarrow \infty} \frac{\operatorname{Pr}(N>(1-\varepsilon) x / \mu)}{\operatorname{Pr}(N>x / \mu)}=1 .
$$


To establish the asymptotic lower bound for $\operatorname{Pr}\left(S_{N}>x\right)$, with arbitrarily fixed $0<\varepsilon<1$ we derive

$$
\begin{aligned}
\operatorname{Pr}\left(S_{N}>x\right) & \geq \sum_{n>(1+\varepsilon) x / \mu} \operatorname{Pr}\left(S_{n}>x\right) \operatorname{Pr}(N=n) \\
& \geq \sum_{n>(1+\varepsilon) x / \mu} \operatorname{Pr}\left(\frac{1}{n} S_{n}-\mu>-\frac{\varepsilon \mu}{1+\varepsilon}\right) \operatorname{Pr}(N=n) \\
& \sim \operatorname{Pr}\left(N>\frac{(1+\varepsilon) x}{\mu}\right),
\end{aligned}
$$

where in the last step we used the fact that, by Theorem 1.1,

$$
\lim _{x \rightarrow \infty} \sup _{n>(1+\varepsilon) x / \mu}\left|\operatorname{Pr}\left(\frac{1}{n} S_{n}-\mu>-\frac{\varepsilon \mu}{1+\varepsilon}\right)-1\right| .
$$

It follows that

$$
\liminf _{x \rightarrow \infty} \frac{\operatorname{Pr}\left(S_{N}>x\right)}{\operatorname{Pr}(N>x / \mu)} \geq \lim _{\varepsilon \rightarrow 0+} \liminf _{x \rightarrow \infty} \frac{\operatorname{Pr}(N>(1+\varepsilon) x / \mu)}{\operatorname{Pr}(N>x / \mu)}=1 .
$$

A combination of (4.7) and (4.8) gives the desired asymptotic relation (4.3).

\subsection{Application to renewal theory}

Let $\left\{N_{t}, t \geq 0\right\}$ be a quasi-renewal counting process defined as

$$
N_{t}=\max \left\{n=1,2, \ldots: \sum_{k=1}^{n} Y_{k} \leq t\right\}, \quad t \geq 0,
$$

where the interarrival times $Y_{k}, k=1,2, \ldots$, form a sequence of nonnegative, END, and identically distributed random variables with common distribution $G$ and finite, positive mean $1 / \lambda$.

Theorem 4.2. Consider the quasi-renewal counting process defined by (4.9). As $t \rightarrow \infty$,

(a) $N_{t} /(\lambda t) \stackrel{\text { a.s. }}{\rightarrow} 1$;

(b) $\mathrm{E} N_{t}^{p} \sim(\lambda t)^{p}$ for every $p>0$.

Proof. (a) We follow the proof of Proposition 5.1.4 of Asmussen (2003). By Theorem 1.1(b),

$$
\lim _{n \rightarrow \infty} \frac{1}{n} \sum_{k=1}^{n} Y_{k} \stackrel{\text { a.s. }}{=} \frac{1}{\lambda}
$$

It trivially follows that $N_{t} \stackrel{\text { a.s. }}{\rightarrow} \infty$ as $t \rightarrow \infty$. Note that

$$
\sum_{k=1}^{N_{t}} Y_{k} \leq t<\sum_{k=1}^{N_{t}+1} Y_{k}
$$

Dividing each side of the above by $N_{t}$, then letting $t \rightarrow \infty$, we obtain $t / N_{t} \stackrel{\text { a.s. }}{\rightarrow} 1 / \lambda$. 
(b) Following the proof of Theorem 1 of Kočetova et al. (2009) with some modifications in relation to the idea used in deriving (2.6), we have, for every $a>1$ and some $b>1$,

$$
\lim _{t \rightarrow \infty} \sum_{n>a \lambda t} b^{n} \operatorname{Pr}\left(N_{t} \geq n\right)=0 .
$$

Split the moment $\mathrm{E} N_{t}^{p}$ into two parts as

$$
\mathrm{E} N_{t}^{p}=\left(\sum_{1 \leq n \leq a \lambda t}+\sum_{n>a \lambda t}\right) n^{p} \operatorname{Pr}\left(N_{t}=n\right)=I_{1}(t)+I_{2}(t) .
$$

By the dominated convergence theorem,

$$
\lim _{t \rightarrow \infty} \frac{I_{1}(t)}{(\lambda t)^{p}}=\lim _{t \rightarrow \infty} \mathrm{E}\left(\frac{N_{t}}{\lambda t}\right)^{p} \mathbf{1}_{\left\{1 \leq N_{t} \leq a \lambda t\right\}}=1 .
$$

By (4.10), as $t \rightarrow \infty$,

$$
I_{2}(t)=o(1) \sum_{n>a \lambda t} b^{n} \operatorname{Pr}\left(N_{t} \geq n\right)=o(1) .
$$

Substituting these estimates into (4.11) leads to $\mathrm{E} N_{t}^{p} \sim(\lambda t)^{p}$ as $t \rightarrow \infty$.

\section{References}

AlešKeVIČIENĖ, A., LePUS, R. AND ŠIAUlys, J. (2008). Tail behavior of random sums under consistent variation with applications to the compound renewal risk model. Extremes 11, 261-279.

Asmussen, S. (2003). Applied Probability and Queues, 2nd edn. Springer, New York.

BAEK, J.-I., SEO, H.-Y., LeE, G.-H. AND ChOI, J.-L. (2009). On the strong law of large numbers for weighted sums of arrays of rowwise negatively dependent random variables. J. Korean Math. Soc. 46, 827-840.

Bingham, N. H. AND NILI SANI, H. R. (2004). Summability methods and negatively associated random variables. In Stochastic Methods and Their Applications (J. Appl. Prob. 41A), eds J. Gani and E. Seneta, Applied Probability Trust, Sheffield, pp. 231-238.

Block, H. W., Savits, T. H. And Shaked, M. (1982). Some concepts of negative dependence. Ann. Prob. 10, 765-772.

Chen, Y., Yuen, K. C. AND NG, K. W. (2010). Precise large deviations of random sums in presence of negative dependence and consistent variation. To appear in Methodology Comput. Appl. Prob. .

Cossette, H., Marceau, E. ANd Marri, F. (2008). On the compound Poisson risk model with dependence based on a generalized Farlie-Gumbel-Morgenstern copula. Insurance Math. Econom. 43, 444-455.

Denisov, D., Foss, S. AND Korshunov, D. (2010). Asymptotics of randomly stopped sums in the presence of heavy tails. Bernoulli 16, 971-994.

Ebrahimi, N. ANd GHosh, M. (1981). Multivariate negative dependence. To appear in Commun. Statist. Theory Meth. 10, 307-337.

Gerasimov, M. Yu. (2009). The strong law of large numbers for pairwise negatively dependent random variables. Moscow Univ. Comput. Math. Cybernet. 33, 51-58.

Hashorva, E. (2001). Asymptotic results for FGM random sequences. Statist. Prob. Lett. 54, 417-425.

Joe, H. (1997). Multivariate Models and Dependence Concepts. Chapman and Hall, London.

Ko, B. And TANG, Q. (2008). Sums of dependent nonnegative random variables with subexponential tails. J. Appl. Prob. 45, 85-94.

KočEtova, J., Leipus, R. AND ŠIAUlys, J. (2009). A property of the renewal counting process with application to the finite-time ruin probability. Lithuanian Math. J. 49, 55-61.

Kochen, S. And Stone, C. (1964). A note on the Borel-Cantelli lemma. Illinois J. Math. 8, 248-251.

Kotz, S., Balakrishnan, N. And Johnson, N. L. (2000). Continuous Multivariate Distributions, Vol. 1, 2 nd edn. Wiley-Interscience, New York.

LiU, L. (2009). Precise large deviations for dependent random variables with heavy tails. Statist. Prob. Lett. 79, 12901298.

MatuŁA, P. (1992). A note on the almost sure convergence of sums of negatively dependent random variables. Statist. Prob. Lett. 15, 209-213. 
Nelsen, R. B. (2006). An Introduction to Copulas, 2nd edn. Springer, New York.

Robert, C. Y. AND SEgERs, J. (2008). Tails of random sums of a heavy-tailed number of light-tailed terms. Insurance Math. Econom. 43, 85-92.

TANG, Q. (2006). Insensitivity to negative dependence of the asymptotic behavior of precise large deviations. Electron. J. Prob. 11, 107-120.

TANG, Q. And Tsitsiashvili, G. (2003). Precise estimates for the ruin probability in finite horizon in a discrete-time model with heavy-tailed insurance and financial risks. Stoch. Process. Appl. 108, 299-325.

TANG, Q. AND Vernic, R. (2007). The impact on ruin probabilities of the association structure among financial risks. Statist. Prob. Lett. 77, 1522-1525.

TANG, Q. AND YAN, J. (2002). A sharp inequality for the tail probabilities of sums of i.i.d. r.v.'s with dominatedly varying tails. Sci. China Ser. A 45, 1006-1011.

YAN, J. (2006). A simple proof of two generalized Borel-Cantelli lemmas. In Memoriam Paul-André Meyer: Séminaire de Probabilités XXXIX (Lecture Notes Math. 1874), Springer, Berlin, pp. 77-79. 